\title{
Quantum Monte Carlo Simulations of Disordered Magnetic and Superconducting Materials
}

\author{
R.T. Scalettar, P.J.H. Denteneer, C. Huscroft, A. McMahan,
}

R. Pollock, M. Randeria, N. Trivedi, M. Ulmke and G.T. Zimanyi

This paper was prepared for submittal to the

Materials Research Society 1997 Fall Meeting

Boston, $M A$

December 1-5, 1997

January 1998

This is a preprint of a paper intended for publication in a journal or proceedings. Since changes may be made before publication, this preprint is made available with the understanding that it will not be cited or reproduced without the permission of the author. 


\section{DISCLAIMER}

This document was prepared as an account of work sponsored by an agency of the United States Government. Neither the United States Government nor the University of California nor any of their employees, makes any warranty, express or implied, or assumes any legal liability or responsibility for the accuracy, completeness, or usefulness of any information, apparatus, product, or process disclosed, or represents that its use would not infringe privately owned rights Reference herein to any specific commercial product, process, or service by trade name, trademark, manufacturer, or otherwise, does not necessarily constitute or imply its endorsement, recommendation, or favoring by the United States Government or the University of California The views and opinions of authors expressed herein do not necessarily state or reflect those of the United States Government or the University of California, and shall not be used for advertising or product endorsement purposes 


\title{
QUANTUM MONTE CARLO SIMULATIONS OF DISORDERED MAGNETIC AND SUPERCONDUCTING MATERIALS
}

\author{
R.T.-SCALETTAR ${ }^{1}$, P.J.H. DENTENEER ${ }^{2}$, C. HUSCROFT ${ }^{1}$, A. MCMAHAN ${ }^{3}$, \\ R. POLLOCK ${ }^{3}$, M. RANDERIA ${ }^{4}$, N. TRIVEDI ${ }^{4}$, M. ULMKE $^{5}$, G.T. ZIMANYI ${ }^{1}$ \\ ${ }^{1}$ Physics Department, University of California, Davis, CA 95616 \\ ${ }^{2}$ Lorentz Institute, Leiden University, 2333 CA Leiden, The Netherlands \\ ${ }^{3}$ Lawrence Livermore National Laboratory P. O. Box 808, Livermore, CA 94551 \\ ${ }^{4}$ Theor. Phys. Group, Tata Institute of Fundamental Research, Bombay 400005, India \\ ${ }^{5}$ Theor. Phys. III, Institut für Physik, Universität Augsburg, D-86135 Augsburg, Germany
}

\section{ABSTRACT}

Over the last decade, Quantum Monte Carlo (QMC) calculations for tight binding Hamiltonians like the Hubbard and Anderson lattice models have made the transition from addressing abstract issues concerning the effects of electron-electron correlations on magnetic and metal-insulator transitions, to concrete contact with experiment. This paper presents results of applications of "determinant" QMC to systems with disorder such as the conductivity of thin metallic films, the behavior of the magnetic susceptibility in doped semiconductors, and Zn doped cuprate superconductors. Finally, preliminary attempts to model the Kondo volume collapse in rare earth materials are discussed.

\section{INTRODUCTION}

The determinant QMC method[1] is a powerful technique for understanding the physics of itinerant, interacting electrons. Its primary strength is that it treats the correlations between electrons exactly, in contrast to other approaches which resort to various simplifying approximations. The chief disadvantage is its computational cost, which limits the complexity of the models which can be considered. Many of the past applications[2] have been to the single-band two-dimensional Hubbard Hamiltonian. This model is of theoretical interest since it is the simplest lattice Hamiltonian exhibiting both an interaction-driven ("Mott") metal-insulator transition and also long range magnetic order. It is also potentially of importance in understanding the magnetic and superconducting properties of the $\mathrm{CuO}_{2}$ sheets of high temperature superconductors. That the model has a single band and is in two-dimensions has played a crucial role in making simulations on reasonable lattice sizes (up to $16 \times 16$ sites) possible.

As algorithms and machine speeds have improved, however, computational restrictions are becoming less prohibitive, and the determinant QMC approach is being applied to tight-binding models which include features such as many orbitals, disorder, and higher dimensionality. In this paper we will provide an overview of four such applications. First, we have studied a model of disordered superconducting films, where there has been a long-standing interest in the possibility of a universal conductivity at the superconductorinsulator phase transition. [3] Second, we examine the effect of topological randomness on magnetically ordered phases, where one issue is the enhancement of the uniform spin susceptibility at low temperatures observed in doped semiconductors.[4] We next describe the behavior of magnetic correlations when non-magnetic impurity sites are introduced, a problem under investigation with recent experiments on $\mathrm{Zn}$ doping of ladder compounds 
and high temperature superconductors.[5] Finally, we describe preliminary results on the phase diagram of the periodic Anderson Hamiltonian in three dimensions.[6] Here the key question is constructing a minimal model which might contain the essential features necessary for describing the "volume-collapse" transition observed in many rare earth systems.

In the remainder of this introduction, we will describe in more detail the physics of these problems. Following this, section 2 contains a discussion of the QMC algorithm. Sections 3,4 , and 5 contain the results of our simulations, and section 6 is a brief conclusion.

i. Most bulk metals, and many alloys, undergo a superconducting phase transition in which the resistivity plunges abruptly to zero as the temperature is lowered. Over the last two decades, this transition has been exhaustively studied for thin films. [7] When small amounts of $\mathrm{Pb}$ or $\mathrm{Sn}$, for example, are deposited on a substrate, the resistance increases as the temperature is lowered; the material is an insulator. However, as more miaterial is deposited, the resistance goes to zero as the temperature is lowered. That is, there is a superconducting-insulator transition controlled by the film thickness. These experiments have been carried out for a large variety of materials and substrates, and were also performed with different tuning parameters for the transition, for example changing the magnetic field strength in $\mathrm{Bi}$ or oxygen concentration in $\mathrm{In}_{1-x} \mathrm{O}_{x}$ samples at fixed thickness.

The remarkable observation is that the "critical resistance" which separates systems which are insulating from those which are superconducting is nearly the same for all materials. This is, of course, surprising, since such universality is normally expected in quantities like critical exponents, not in the coupling strength determining the location of a transitions. There have been a number of analytic attempts to explain this phenomenon, [8] but until very recently numeric approaches have been restricted to simplified "boson" models in which the electrons are treated as preformed Cooper pairs. [8, 9]

In section 3 we describe QMC calculations for a model of interacting, two-dimensional electrons which is known to display a superconducting phase transition as the temperature is lowered. We add disorder to this model, and show that a superconductor-insulator phase transition is induced, and evaluate the resistance at the transition.

ii. Doped semiconductors like Si:P exhibit a number of interesting magnetic phenomena. The basic physics is that of a two-fluid system with both itinerant electrons and randomly positioned local moments.[10] One essential question concerns whether there is a low temperature divergence of the magnetic susceptibility $\chi(q=0)$. It has been suggested that random exchange couplings between moments can lead to isolated spins which have a Curie-like contribution to $\chi$. Similar rapid increases in the susceptibility as the temperature is decreased are observed in the quasi-1D TCNQ compounds,[11] and are likewise believed to have their origin in randomness. Section 4a contains a discussion of simulations of a model of itinerant interacting electrons with hopping disorder which captures this behavior.

Another magnetic system in which controlled disorder can be studied is $\mathrm{Zn}$ substitution in ladder compounds[12] and in the cuprate superconductors.[13] There it is observed that antiferromagnetic order can be stabilized by randomness. Recent numerical studies have shown that, indeed, short range magnetic correlations can be enhanced by non-magnetic defects.[14] Here we present, in section 4b, a discussion of the effect of non-magnetic impurities on long range magnetic correlations and on the Mott metal-insulator transition.

iii. Many rare earth systems undergo a transition in which the volume is abruptly and drastically reduced as the pressure increases. For example, Ce, a particularly simple 
casc because the transition is isostructural, exhibits a first order phase transition with a $15 \%$ change in volume at $8 \mathrm{kbar}$. There are a number of competing theories for the origin of this effect.[15, 16] At the crudest level, a transfer of electrons between localized $f$ and itinerant $d$ orbitals might account for the volume change. Many theories assume that electron correlations play a crucial role, because the transition involves a loss of magnetic moments.

In section 5, we describe QMC simulations of the three-dimensional periodic Anderson model (PAM). This Hamiltonian has the requisite complexity to allow for the processes which may underly the volume collapse transition, namely separate conduction and localized orbitals and the potential for charge transfer between them; and also the possibility of "singlet" formation between electron spins in the two orbitals which would account for the disappearance of magnetic moments.

\section{THE DETERMINANT QUANTUM MONTE CARLO METHOD}

Determinant QMC has proven very useful for evaluating correlation functions in tightbinding problems of interacting electrons. Consider the Hubbard Hamiltonian,

$$
\hat{H}=-\sum_{\langle\mathbf{i}, \mathbf{j}\rangle \sigma} t_{\mathbf{i j}}\left(c_{\mathbf{i} \sigma}^{\dagger} c_{\mathbf{j} \sigma}+c_{\mathbf{j} \sigma}^{\dagger} c_{\mathbf{i} \sigma}\right)+\sum_{\mathbf{i}}\left[U_{\mathbf{i}}\left(n_{\mathbf{i} \uparrow}-\frac{1}{2}\right)\left(n_{\mathbf{i} \downarrow}-\frac{1}{2}\right)-\mu_{\mathbf{i}}\left(n_{\mathbf{i} \uparrow}+n_{\mathbf{i} \downarrow}\right)\right]
$$

which consists of a kinetic energy term describing the hopping of electrons between nearestneighbor sites $\mathbf{i}$ and $\mathbf{j}$, an interaction term giving a repulsion between electrons of opposite spin species on the same site, and a chemical potential term which controls the filling.[17] Since our interest will be in disordered models, we have explicitly indicated that the transfer integrals $t_{\mathbf{i j}}$, interaction strengths $U_{\mathbf{i}}$, and local chemical potentials $\mu_{\mathbf{i}}$ may be random. The Hamiltonian Eq. 1 is "particle-hole symmetric" at $\mu_{\mathbf{i}}=0$ so that each site is precisely "halffilled", $\left\langle n_{\mathbf{i}}\right\rangle=1$, regardless of the values of the hopping and interactions (in the absence of charge-density-wave formation).

In general, QMC methods map a quantum problem in $d$ spatial dimensions to a classical problem in $d+1$ dimensions, where the inverse temperature $\beta=1 / T$ gives the additional length to the lattice. The reason is that in a classical statistical mechanics problem, the Boltzmann factor, $\exp [-\beta E]$, is a real-valued function of the degrees of freedom. In a quantum mechanical problem the relevant quantity is $\exp [-\beta \hat{H}]$ where $\hat{H}$ is an operator.

This exponential of the Hamiltonian operator cannot be computed without diagonalizing $\hat{H}$ (that is, solving the problem exactly). However, it is often the case that the exponential of the kinetic and potential energy pieces of $\hat{H}$ can be separately diagonalized, for example by working in momentum- or real- space respectively. Unfortunately, these constituent pieces do not commute, so the full exponential cannot be broken up into the product of the individual exponentials. The trick is to discretize the inverse temperature $\beta$ into a large number $L$ of much smaller divisions $\Delta \tau=\beta / L$ and use the "Trotter approximation,"[18]

$$
Z=\operatorname{Tr}\left[e^{-\beta \hat{H}}\right]=\operatorname{Tr}\left[e^{-\Delta \tau \hat{H}}\right]^{L} \approx \operatorname{Tr}\left[e^{-\Delta \tau \hat{K}} e^{-\Delta \tau \hat{V}}\right]^{L} .
$$

The discretization of $\beta$ has introduced a small parameter and breaking up the exponential is now a procedure which becomes arbitrarily accurate as $L$ increases. This discretization of $\beta$ generates the added "inverse temperature" dimension to quantum simulations. In the 
case of the Hubbard Hamiltonian, $\hat{K}$ includes the one body terms, the kinetic energy and chemical potential, and $\hat{V}$ includes the two-body interactions.

In the "world-line" algorithm, complete sets of states are introduced into Eq. 2, and the resulting matrix elements are evaluated analytically. The determinant QMC algorithm instead reduces the exponential of $\hat{V}$ to an exponential of a one body operator by introducing an integration over an auxiliary "Hubbard-Stratonovich" ficld,

$$
e^{-\Delta \tau U\left(n_{\mathbf{i} \uparrow}-\frac{1}{2}\right)\left(n_{\mathbf{i} \downarrow}-\frac{1}{2}\right)}=\frac{1}{2} e^{-\wedge \tau U / 4} \sum_{S(\mathbf{i}, l)= \pm 1} e^{-\lambda S(\mathbf{i}, l)\left(n_{\mathbf{i} \uparrow}-n_{\mathbf{i} \downarrow}\right)}
$$

The coupling constant $\lambda$ is given by $\cosh (\lambda)=\exp [\Delta \tau U / 2]$. We must introduce a field variable $S(\mathbf{i}, l)$ at each spatial site $\mathbf{i}$ of the lattice where an interaction is present, and also at each imaginary time slice $l$ in the Trotter decomposition of $\exp [-\beta \hat{H}]$.

The final step is to evaluate the trace over the fermion degrees of freedom, which can now be performed analytically since the exponentials involve only one-body terms.

$$
Z=\sum_{\{S(\mathbf{i}, l)\}= \pm 1} \operatorname{det} M_{\uparrow}(S) \operatorname{det} M_{\downarrow}(S) .
$$

Detailed forms for the matrices $M_{\sigma}$, which have dimension the number of spatial sites in the lattice, are given in the literature.[1] To summarize Eq. 4, the quantum mechanical partition function has been expressed in terms of an equivalent classical problem in one higher dimension; $Z$ is a sum over a classical field indexed by $\mathbf{i}$ and $l$ with an argument which no longer involves operators but is instead an effective classical Boltzmann weight.

We conclude with some general comments. First, the scaling of the algorithm is as the cube of the number of sites,[19] because of computations of the determinants of the matrices $M_{\sigma}$. Simulations of about 100 sites are already fairly computationally intensive, indicating why most applications have been to the simplest single orbital models in low dimension. Second, we are actually interested in measuring operator expectation values, $\langle\hat{A}\rangle=Z^{-1} \operatorname{Tr}\left[\hat{A} e^{-\beta \hat{H}}\right]$. Here, $\hat{A}$ might be the charge, spin, superconducting pair, or current density on a site or a "correlation function" consisting of a product of two such densities on different sites of the lattice. Such traces can easily be formulated in the same way as outlined above for $Z$, and, indeed, measurements of such physical quantities involve only accumulating the appropriate elements of the inverse of $M_{\sigma}$. Finally, there is a very important limitation to determinant QMC simulations. The determinants can become negative, and in such cases measurements can have impractically large variance. In this paper we will deal with situations where this problem does not occur.[20]

The calculations presented here will be mainly results from determinant QMC, but we will also show a few computations using "dynamical mean-field theory" (DMFT).[21] This technique also employs an auxiliary-field QMC algorithm, but introduces a local approximation to the self-energy, which becomes exact in the limit of infinite dimensions, and allows a more complete exploration of parameter space.

\section{DISORDER-DRIVEN SUPERCONDUCTOR-INSULATOR PHASE TRANSITIONS}

We first study a Hamiltonian of the form given in Eq. 1 with uniform hoppings $t_{\mathrm{ij}}=t$ and uniform attractive interactions $U_{\mathbf{i}}=-|U|$. In the absence of randomness in the chemical potential, this "attractive Hubbard model" is known to exhibit a KosterlitzThouless phase transition to a superconducting state as the temperature is lowered off half-filling. 

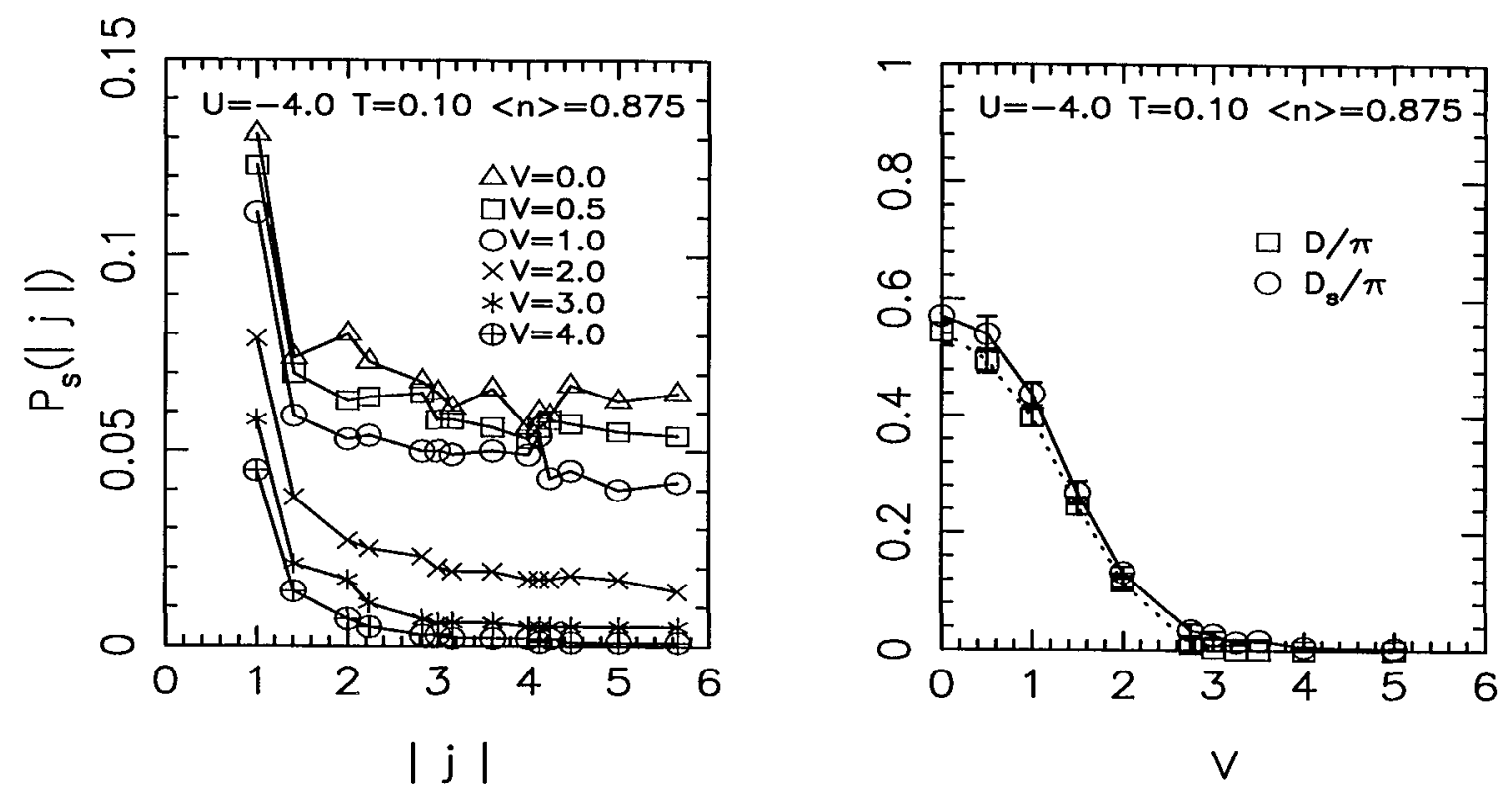

Figure 1: Left panel: Pairing correlations as a function of lattice separation for different disorder strengths, $-V / 2<\mu_{\mathrm{i}}<V / 2$. For $V \approx 3$ the correlations become very small at large distances. Right panel: Superfluid stiffness $D_{s}$ as a function of disorder strength. Again, for $V \approx 3$ this measure of superconductivity vanishes. The Drude weight $D$, the coefficient of $\delta(\omega)$ in the ac conductivity, $\sigma(\omega)$, is also shown, and equals $D_{s}$ for this system. The lattice size is $8 \times 8$.

In Fig. 1 we show what happens to two quantities which measure whether the system is in a superconducting state. The pair correlation function,

$$
p_{s}(\mathbf{j})=\left\langle\Delta_{\mathbf{i}+\mathbf{j}} \Delta_{\mathbf{i}}^{\dagger}\right\rangle \quad \Delta_{\mathbf{j}}^{\dagger}=c_{\uparrow \mathbf{j}}^{\dagger} c_{\downarrow \mathbf{j}}^{\dagger},
$$

will go to a constant non-zero value at large separations $\mathbf{j}$ if the state is superconducting, and otherwise decay to zero.

The superfluid stiffness is a second quantity which is non-zero in the superconducting state. It is defined in terms of an appropriate limit of the current-current correlation function,

$$
\begin{aligned}
D_{s} & =-K_{x}-\Lambda^{\mathrm{T}}, \\
K_{x} & =\left\langle-t \sum_{\sigma}\left(c_{1+\hat{x}, \sigma}^{\dagger} c_{1, \sigma}+c_{1, \sigma}^{\dagger} c_{1+\hat{x}, \sigma}\right)\right\rangle, \\
\Lambda^{T} & \equiv \lim _{q_{y} \rightarrow 0} \Lambda_{x x}\left(q_{x}=0, q_{y} ; i \omega_{n}=0\right), \\
\Lambda_{x x}\left(\mathbf{q} ; i \omega_{n}\right) & =\sum_{1} \int_{0}^{\beta} d \tau\left\langle j_{x}(\mathbf{1}, \tau) j_{x}(0,0)\right\rangle e^{i \mathbf{q} \cdot \mathbf{1}} e^{-i \omega_{n} \tau}, \\
j_{x}(\mathbf{1}, \tau) & =e^{H \tau}\left[i t \sum_{\sigma}\left(c_{\mathbf{1}+\hat{x}, \sigma}^{\dagger} c_{1, \sigma}-c_{1, \sigma}^{\dagger} c_{\mathbf{1}+\hat{x}, \sigma}\right)\right] e^{-H \tau} .
\end{aligned}
$$

Here $\omega_{n}=2 n \pi / \beta$. In the right panel of Fig. 1 we show $D_{s}$ as a function of the randomness $V$ in the chemical potential. Disorder drives a transition to a state in which the pairing order is destroyed and the superfluid stiffness vanishes. 

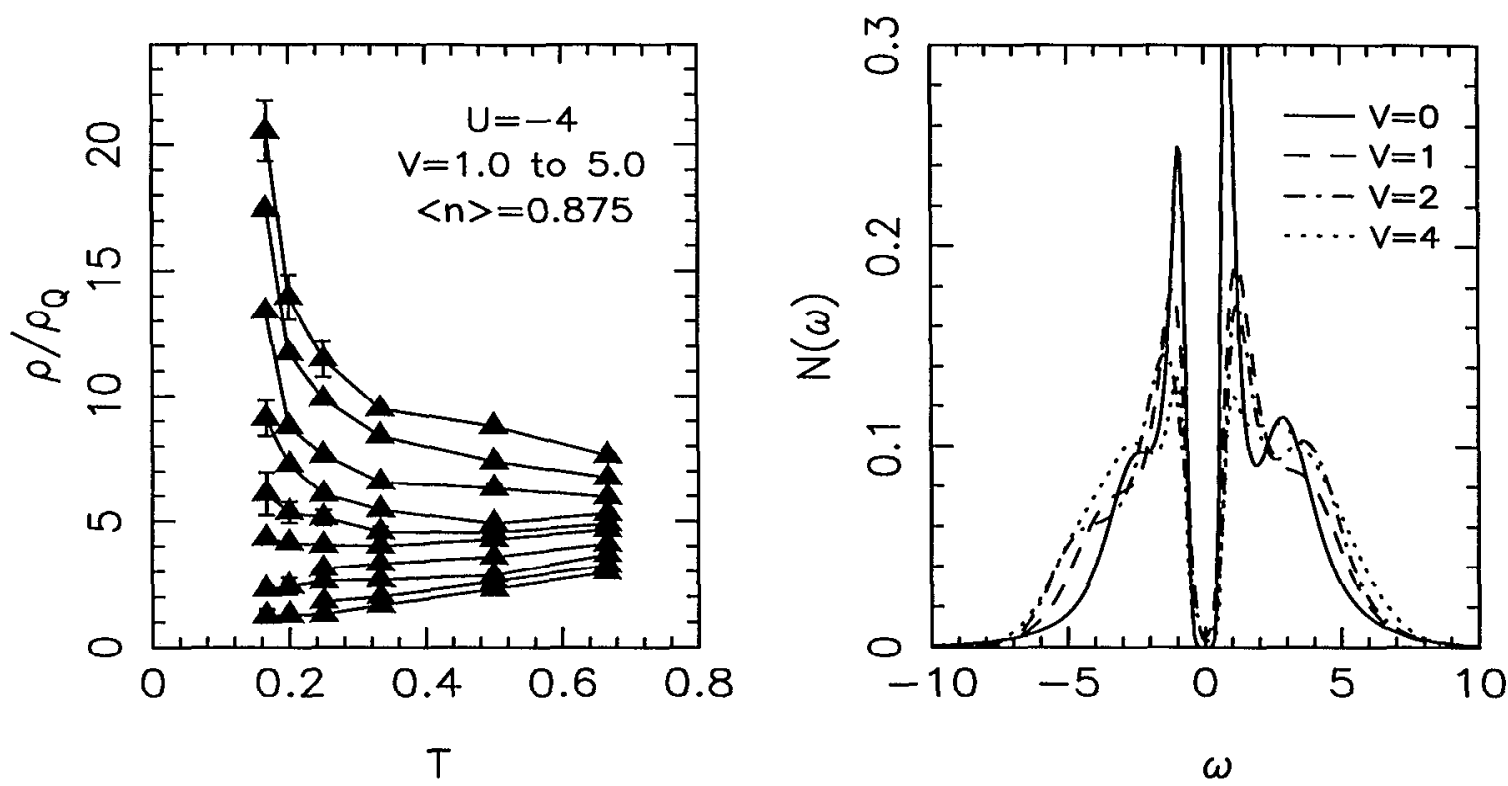

Figure 2: Left panel: dc resistivity as a function of temperature for different disorder strengths. For $V \approx 3$ the traces show a transition from superconducting $(d \rho / d T>0)$ to insulating $(d \rho / d T<0)$ behavior. $\rho_{Q}=\hbar^{2} / 4 e^{2}$ is the quantum of resistance. Right panel: Density of states $N(\omega)$ as we sweep through the transition. For both panels, the lattice size is $8 \times 8$.

What happens to the resistivity, $\rho_{\mathrm{dc}}$, the experimentally most interesting quantity, as we cross through this transition? In the left panel of Fig. 2 we show $\rho_{\mathrm{dc}}$ as a function of temperature. This plot looks remarkably similar to that seen in experiments[7]: a family of traces exhibiting insulating behavior is separated from a family where the resistivity decreases as $T$ is lowered, with a roughly constant separating curve at a critical disorder strength. (This value is consistent with estimates of the transition point obtained from the data of Fig. 1.) The value of the critical resistance is roughly the same as that obtained in previous numerical studies of bosonic models, which are the $|U| / t \rightarrow \infty$ limit of the Hamiltonian considered here. $[8,9]$ This value is about twice that observed experimentally, a disagreement which may be due to the lack of long range Coulomb repulsion in the model. We are currently studying this issue in greater detail.

'I'he right panel of Fig. 2 shows the density of states (DOS). Interestingly, the gap in the density of states which exists in the superconducting phase is not destroyed when the disorder is increased to large enough values to suppress pair correlations and $D_{s}$ (Fig. 1) to zero. This is in contrast to results[22] for the repulsive Hubbard model in which the analogous long range AF correlations appear to be necessary for the existence of a gap in $N(\omega)$. The DOS was obtained using the "maximum entropy algorithm." [23] Our results show that this technique works well even in the presence of disorder and the associated error bars from disorder averaging.

\section{DISORDER-DRIVEN MAGNETIC PHASE TRANSITIONS}

In the previous section we saw that random chemical potentials drive a superconductinginsulator transition and evaluated the resistivity at the transition, a quantity which exhibits 
a roughly "universal" value experimentally. Just as disorder interferes with long range pairing order in the attractive Hubbard model, it also can affect magnetic correlations in the repulsive case. In this section we describe simulations of these effects in models which have been suggested as appropriate to $\mathrm{P}$ doped $\mathrm{Si}$, and $\mathrm{Zn}$ doped $\mathrm{LaCuO}_{4}$.

\section{Random Bond Models}

It is believed that the random hopping Hubbard Hamiltonian, and its strong coupling limit the random exchange Heisenberg Hamiltonian, might be appropriate models of the behavior of the magnetic susceptibility in doped semiconductors.[25] The idea is that as the randomness is increased, singlets can form on strong bonds, instead of the magnetically ordered phase in which all spins are correlated, which is the ground state in the clean limit. Depending on the topology, some single spins may be left isolated in this process of singlet formation, and these spins contribute a divergent Curie term to the magnetic susceptibility at low temperatures. While the Hubbard case has been studied analytically, and numerically on small lattices, QMC simulations in two dimensions have only recently been reported.[4] Here we review simulations of Eq. 1 in which the interactions $U_{\mathbf{i}}=U>0$ and chemical potentials $\mu_{\mathrm{i}}=0$ are constant, but the hopping is disordered, $t-\Delta / 2<t_{\mathrm{ij}}<$ $t+\Delta / 2$. We show that indeed, anomalies in $\chi$ do appear.

The left panel of Fig. 3 shows the spin-spin correlation function,

$$
C(\mathbf{l})=\left\langle M_{\mathbf{i}+\mathbf{1}}^{z} M_{\mathbf{i}}^{z}\right\rangle=\left\langle\left(n_{\mathbf{i}+\mathbf{l} \uparrow}-n_{\mathbf{i}+\mathbf{l} \downarrow}\right)\left(n_{\mathbf{i} \uparrow}-n_{\mathbf{i} \downarrow}\right)\right\rangle,
$$

as a function of separation for different disorder strengths. $C(1)$ exhibits a characteristic alternating pattern indicative of antiferromagnetism. Bond disorder steadily suppresses
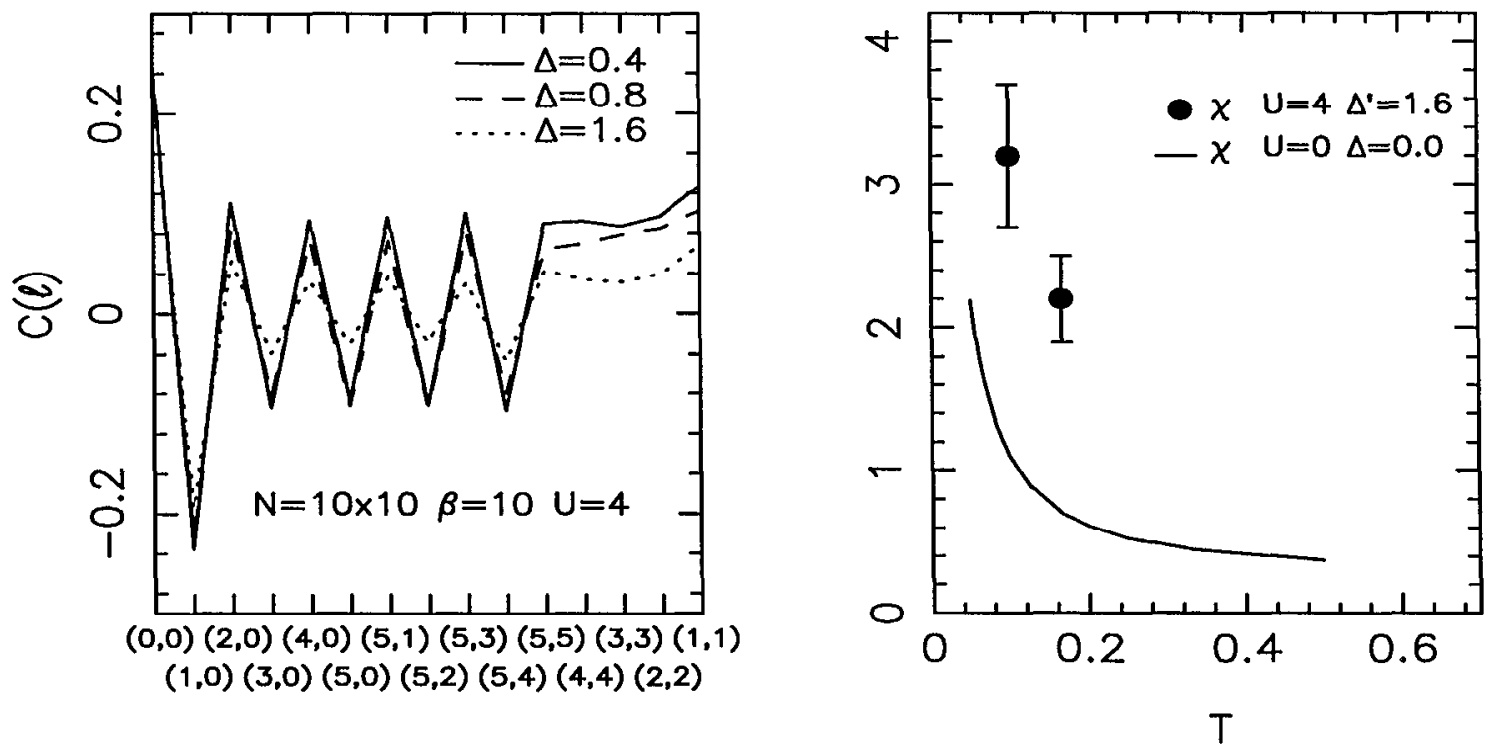

Figure 3: Left panel: Spin correlations as a function of lattice separation for different disorder strengths. For $\Delta \approx 1.6$, a finite size scaling analysis indicates the absence of long range order. Right panel: Uniform susceptibility as a function of temperature. In the presence of disorder and interaction, $\chi$ is enhanced. The "prime" on $\Delta$ indicates the use of a specific type of correlated disorder discussed in Ref. [4]. 
these correlations. A finite size scaling analysis indicates that for $\Delta>\Delta_{c} \approx 1.6$ the AF order parameter vanishes, and, instead, a singlet phase emerges.

The right panel of Fig. 3 exhibits the behavior of the uniform susceptibility,

$$
\chi=\sum_{\mathbf{l}} \int_{0}^{\beta} d \tau\left\langle e^{\tau H} M_{\mathbf{i}+\mathbf{l}}^{z} e^{-\tau H} M_{\mathbf{i}}^{z}\right\rangle,
$$

for different parameter regimes. The solid curve shows the noninteracting limit. In the presence of interactions, and at a small disorder value, $\chi(0,0)$ is reduced below this $U=0$ value. (The AF response, $\chi(\pi, \pi)$ is, of course, dramatically enhanced.) Meanwhile, for $\Delta \approx \Delta_{c}$ the uniform susceptibility instead shows a significant increase.

\section{Nonmagnetic Impurity Models}

We now turn to a model in which the hopping and chemical potential in Eq. 1 are uniform, but the interactions are random. [5] Specifically, we choose to set $U_{\mathbf{i}}$ to zero at some fraction $f$ of the sites of the lattice. One motivation for studying such a model is a purely theoretical one. The Hubbard Hamiltonian at half-filling exhibits both a Mott metalinsulator transition when the energy cost $U$ for double occupancy exceeds the bandwidth, and also AF order. There has recently been considerable discussion concerning whether a Mott gap can open in the absence of symmetry breaking such as that associated with AF order.[24] A model with a fraction $f$ of sites with $U_{\mathbf{i}}=0$ might separate these two phenomena, since the Mott gap will be shifted from half-filling to a density $\langle n\rangle=1+f$, while AF order is likely to remain at half-filling due to commensuration effects.

An experimental motivation for the model is provided by studies of the effect of doping non-magnetic impurities like Zn into ladder compounds and oxide superconductors. One observation is that, in the latter case, AF order persists out to a Zn concentration of $x_{c} \approx 0.10-0.15$, far larger than the amount of doping with an isovalent element like $\mathrm{Sr}$, $x_{c} \approx 0.03$, required to destroy $\mathrm{AF}$.

In Fig. 4 we give results of DMFT simulations of the Hubbard Hamiltonian with $U_{\mathbf{i}}=0$ impurity sites. The left panel shows the phase diagram, and demonstrates the interesting feature that turning on randomness enhances the region of stability of the AF phase. In finite $d$ the region of AF order at zero defect density, $f=0$, will be shrunk down to half-filling $\langle n\rangle=1$, but the enhancement remains and AF order persists significantly away from half-filling. Unfortunately, because of this stabilization of AF order out to the density $\langle n\rangle=1+f$, we conclude this model does not exhibit a separation of Mott and AF behavior.

The right panel of Fig. 4 shows the DOS. At half filling the DOS for the $U=8 t$ sites shows typical AF structure, two broad Hubbard bands, two quasi-particle peaks at low energies, and a gap at the Fermi energy. Surprisingly, there is an induced gap in the AF phase, even for the $U=0$ sites, despite the absence of interactions there. The lower plot shows that at $n=1+f=1.11$, the position of the Mott transition, there is still AF order.

\section{VOLUME COLLAPSE TRANSITIONS}

Several Lanthanides exhibit phase transitions under pressure characterized by abnormally large volume changes (14\% for Cerium and $9 \%$ for Praseodymium). The physical mechanisms responsible for these transitions have been debated since discovery of the Cerium phenomenon over 50 years ago. Currently, the two main viable conjectures are a 

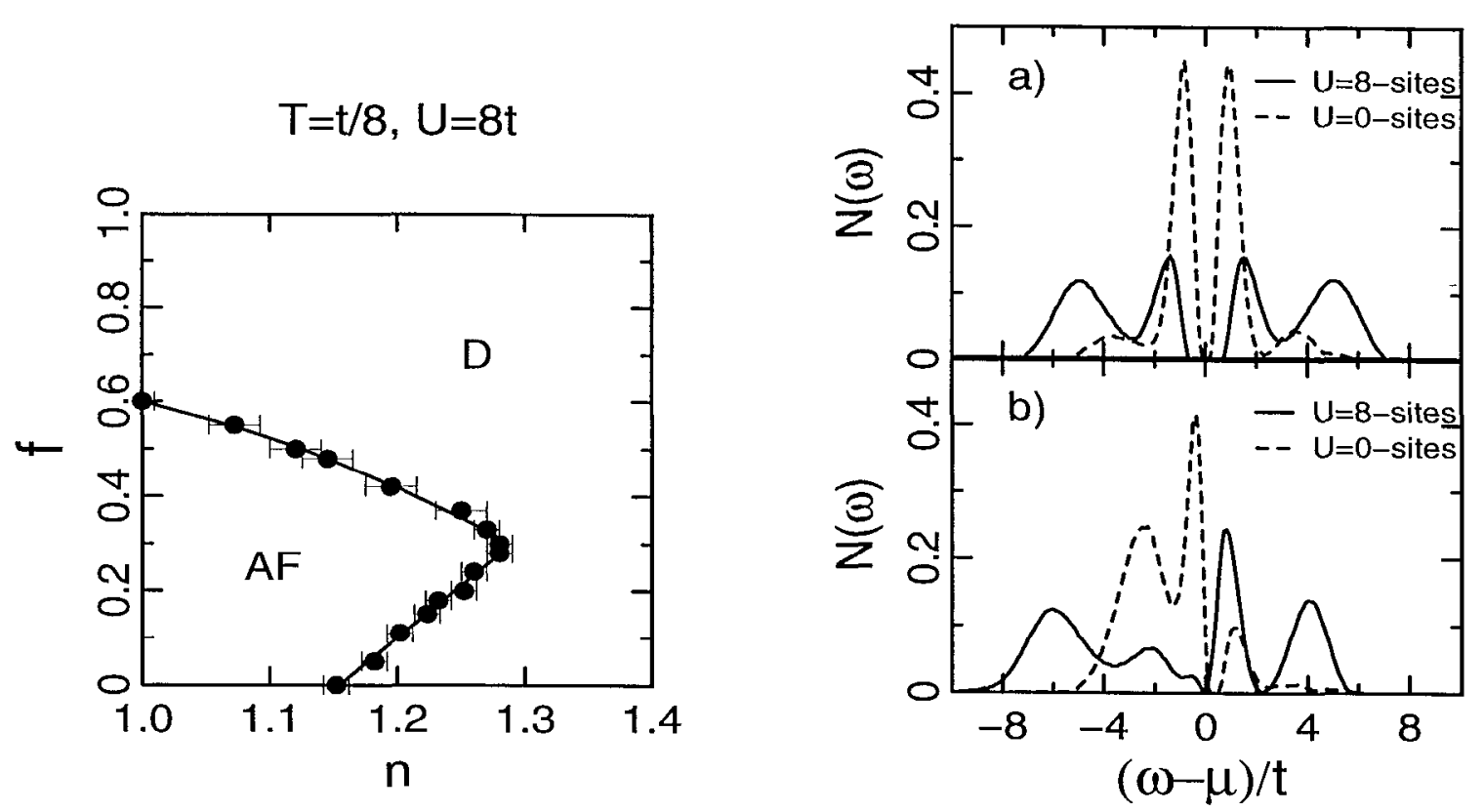

Figure 4: Left panel: Phase diagram of the Hubbard Hamiltonian as a function of filling and concentration of defect sites within dynamical mean field theory. The disorder extends the region of long range AF order to larger doping. A disordered phase (D) is dominant at high carrier and defect doping. Right panel: The density of states on the $U=0$ and $U=8$ sites at a) half-filling and b) $\langle n\rangle=1.11$.

Mott transition of the $4 \mathrm{f}$ electrons accompanied by magnetic ordering[15] and a "Kondo volume collapse" due to rapid change in the 4f-valence electron coupling.[16] In both cases the strongly correlated nature of the $4 \mathrm{f}$ electrons is fundamentally implicated. To model such a transition it is necessary to consider systems with separate conduction and localized orbitals to allow for the transfer of charge between them and also for Kondo screening of the local moments by the conduction electrons. Indeed, our studies of single band models put the transition at grossly incorrect values of the ratio of interaction to bandwidth.[6]

In Fig. 5 we show the phase diagram we have obtained for the three dimensional periodic Anderson model. The precise Hamiltonian we have studied at this point consists of a cubic array of conduction sites hybridized to their near-neighbors by $t_{v v}=1$, and also connected to localized orbitals $\left(t_{f f}=0\right)$, with an inter-orbital hybridization $t_{f v}$. on near-ncighbor sites. We have fixed the repulsion $U_{f f}=8$ on the impurity sites, left the conduction electrons non-interacting, $U_{v v}=0$, and explored the properties of the system as we vary temperature and $t_{f v}$.

We are still in the process of connecting these QMC studies to accurate calculations of the band structure in order to extract thermodynamic behavior and the details of the volume collapse transition. 


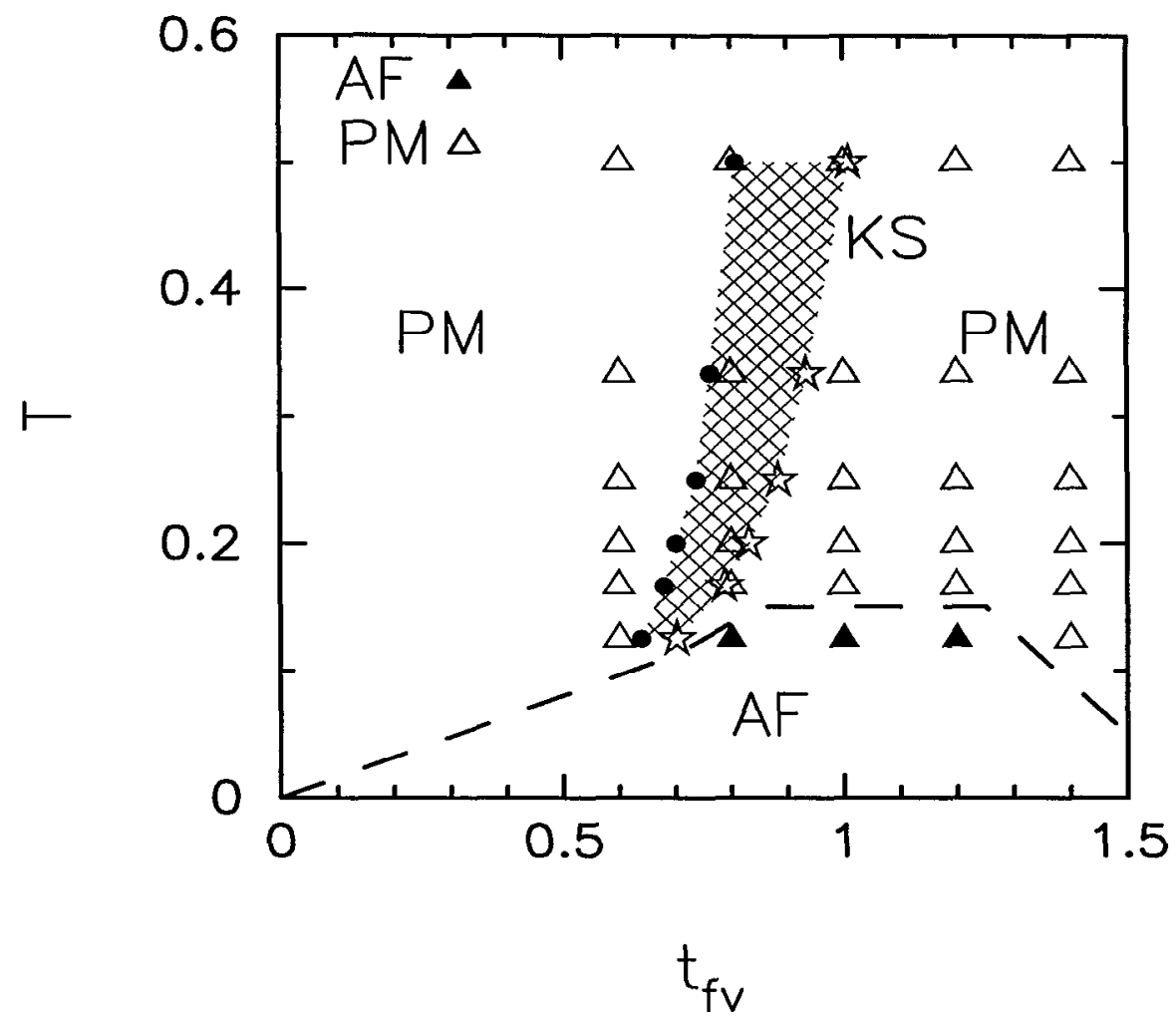

Figure 5: Phase diagram of the periodic Anderson model in three dimensions. At the lowest temperatures the local moments order antiferromagnetically (AF). At higher temperatures there are two distinct disordered regions, separated by a fairly narrow cross-over (hatched area). A paramagnetic (PM) region exists at small $t_{f v}$ and a "Kondo singlet" (KS) region at large $t_{f v}$. These are distinguished by having small (large) values of correlation between near-neighbor $f$ and $d$ electron spins, respectively. The lattice sizes simulated were $4^{3}$ and $6^{3}$.

\section{CONCLUSIONS}

In this paper we have discussed a number of recent determinant QMC simulations of tight-binding Hamiltonians which incorporate some of the complicating features, including disorder, non-trivial orbital structure, and higher dimensionality, which are necessary to describe realistic materials. A significant amount of work remains to be done before these calculations provide compelling descriptions of the experimental properties of solids. While some problems have been solved, for example the extension of maximum entropy methods to obtain dynamical information in disordered systems, significant bottlenecks include adequate treatments of the sign problem to generalize the fillings which are accessible and also to make more practical the study of intersite and interorbital repulsion.

\section{ACKNOWLEDGEMENTS}

We gratefully acknowledge the support of National Science Foundation, grant NSFDMR-9520776 (RTS), the Department of Energy Accelerated Strategic Computing Initiative $(\mathrm{CH})$, and the San Diego Supercomputer Center. 
1. R. Blankenbecler, D.J. Scalapino, and R.L. Sugar, Phys. Rev. D24, 2278 (1981).

2. D.J. Scalapino, Physics Reports 250, 330 (1995); E. Dagotto, Rev. Mod. Phys. 66, 763 (1994).

3. N. Trivedi, R.T. Scalettar, and M. Randeria, Phys. Rev. B54, 3756 (1996).

4. M. Ulmke and R.T. Scalettar, Phys. Rev. B55, 4149 (1997).

5. P. J. H. Denteneer, M. Ulmke, R. T. Scalettar, G. T. Zimanyi, Physica A, to appear. M. Ulmke, P. J. H. Denteneer, R. T. Scalettar, G. T. Zimanyi, unpublished.

6. C. Huscroft, A. McMahan, R.T. Scalettar, work in progress.

7. See review article by A. F. Hebard, in "Strongly Correlated Electronic Systems" edited by K. S. Bedell, 7. Wang, D. F. Meltzer, A. V. Balatsky, and E. Abrahams, (AddisonWesley, 1994).

8. For a brief review, see M. Cha, M.P.A. Fisher, S.M. Girvin, M. Wallin, and A.P. Young, Phys. Rev. B44, 6883 (1991), and the references cited therein.

9. W. Krauth, N. Trivedi and D. M. Cepcrley, Phys. Rev. Lett. 67, 2307 (1991). E. S. Sorensen, M. Wallin, S. M. Girvin, and A. P. Young, Phys. Rev. Lett. 69, 828 (1992). K.J. Runge, Phys. Rev. B45, 13136 (1992). G.G. Batrouni, B. Larson, R.T. Scalettar, J. Tobochnik, and J. Wang, Phys. Rev. B48, 9628 (1993).

10. For a recent review, see D. Belitz and T.R. Kirkpatrick, Rev. Mod. Phys. 66, 261 (1994).

11. L.N. Bulaevskii et.al., Zh. Eksp. Teor. Fiz. 62, p. 725 (1972) [Sov. Phys. JETP 35, 384 (1972)]; and L.J. Azvedo and W.G. Clark, Phys. Rev. B16, 3252 (1977).

12. M. Azuma, Y. Fujishiro, M. Takano, T. Ishida, K. Okuda, M. Nohara, and H. Takagi (to be published); M. Nohara, H. Takagi, M. Azuma, Y. Fujishiro, and M. Takano (to be published).

13. G. Xiao et al., Phys. Rev. Lett. 60, 1466 (1988); B. Kcimer et al., Phys. Rev. B 45, 7430 (1992); A. V. Mahajan, H. Alloul, G. Collin, and J. F. Marucco, Phys. Rev. Lett. 72, 3100 (1994).

14. G.B. Martins, M. Laukamp, J. Riera, and E. Dagotto, Phys. Rev. Lett. 78, 3563 (1997).

15. B. Johansson, Philos. Mag. 30, 469 (1974).

16. J.W. Allen and R.M. Martin, Phys. Rev. Lett. 49, 1106 (1982); and M. Lavagna, C. Lacroix, and M. Cyrot, Phys. Lett. 90A, 210 (1982).

17. While we have used the term "site" for the indices $\mathbf{i}$ and $\mathbf{j}$, these can in fact also label different orbitals. From a technical viewpoint, the determinant QMC algorithm treats distinct sites and orbitals in precisely the same way. They differ only in the Hamiltonian itself, that is in the choice of $t_{\mathbf{i j}}, U_{\mathbf{i}}$, and $\mu_{\mathbf{i}}$. 
18. H.F. Trotter, Proc. Am. Math. Soc. 10, 545 (1959); and M. Suzuki, Phys. Lett. 113A, 299 (1985).

19. Naively the algorithm should scale as the fourth power of the number of sites $N$, since for each move of one of the $N L$ Hubbard-Stratonovich fields, a calculation of the determinant requires $N^{3}$ operations. However, a trick reduces this by one power of $N$.

20. S.R. White, D.J. Scalapino, R.L. Sugar, E.Y. Loh, Jr., J.E. Gubernatis, and R.T. Scalettar, Phys. Rev. B40, 506 (1989).

21. W. Metzner and D. Vollhardt, Phys. Rev. Lett. 62, 324 (1989); for a review see D. Vollhardt, in Correlated Electron Systems, edited by V. J. Emery (World Scientific, Singapore, 1993), p. 57; A. Georges, G. Kotliar, W. Krauth, and M. Rozenberg, Rev. Mod. Phys. 68, 13 (1996); M. Jarrell, Phys. Rev. Lett. 69, 168 (1992); and T. Pruschke, M. Jarrell, and J. K. Freericks, Adv. Phys. 44, 187 (1995).

22. M. Vekic and S.R. White, Phys. Rev. B47, 1160 (1993).

23. M. Jarrell and J. E. Gubernatis, Phys. Rep. 269, 133 (1996).

24. F. Gebhard, The Molt Melal-Insulator Transilion, Springer Tracts in Modern Physics, vol. 137 (Springer, Heidelberg, 1997).

25. M. Milovanovic, S. Sachdev, and R.N. Bhatt, Phys. Rev. Lett. 63, 82 (1989); A.W. Sandvik, D.J. Scalapino, and P. Henelius, Phys. Rev. B50, 10474 (1994); and T.R. Kirkpatrick and D. Belitz, Phys. Rev. Lett. 76, 2571 (1996). 